Pub. Mat. UAB

No 25 Jun; 1981

\title{
Two Coincidence Theorems
}

\author{
Albert Compta Creus \\ Rebut el 16 de Mars del 1981
}

1. INTRODUCTION

This note deals with the existence and unicity of coincidence points $f(x)=g(x)$ for two maps, like in Cerdã $[1]$, related by a contractive type relation similar to the properties required in some generalisations of the Banach fixed point theorem.

In a first theorem we consider maps $f, g$ from a topological space to a complete metric space, following the condition

(i) For each $\varepsilon>0$ there exists $\delta>0$ such that

$$
\varepsilon \leq d(f(x), f(y))<\varepsilon+\delta \Rightarrow d(g(x), g(y))<\varepsilon
$$

like in Meir-Keeler [2]. We shall prove that the coincidence set $S$ is nonemisty and both functions are constant on $S$.

We give an example of mappings keeping contractive relation (i) sut not the one in [1].

In a second theorem based on an article by Chi Song Wong \{3\}, mappings $f, g$ are defined on a uniform space $(X, U)$ and we consider the uniformity basis $U_{\varphi}$ defined by

$$
U_{\varphi}=\left\{\varphi^{-1}(U) \times \varphi^{-1}(U) \cup \Delta: U \in U\right\}
$$

being $\varphi(x)=(\tau(x), g(x))$ and $\Delta$ the diagonal of $X X X$, and we shal i prove that the coincidence set $S$ has a unique point if $f$ is uniformily continuous from 
$\left(x, u_{\varphi}\right)$ into $(x, y)$ and keeps some other conditions.

\section{THEOREM I}

THEOREM 1. Let $X$ be a non-empty topological space and $Y$ a non-empty metric space. Let $f$ and $g$ be two mappings of $X$ into $Y$ keeping (i), and

(ii) $f$ be proper and continuous

(iii) $g(X) \subset f(X)$

(iv) $\overline{g(x)}$ complete.

Then $S$ is non-empty and $f(S)$ has a unique point.

Proof. If $f(x) \neq f(y),(i)$ implies $d(g(x), g(y))<d(f(x), f(y))$ and then $f(S)$ has at great a point.

Now we shall prove that $S$ is non-empty. Let $x_{0} \in X$, (iii) implies $f^{-1}\left\{g\left(x_{0}\right)\right\} \neq \emptyset$. Pick $x_{1} \in f^{-1}\left\{g\left(x_{0}\right)\right\}$. Repeating the same operation we obtain a sequence $x_{n}$ of $x$ keeping $f\left(x_{n+1}\right)=g\left(x_{n}\right)$ for each $n \in N$.

Let $c_{n}=d\left(f\left(x_{n}\right), f\left(x_{n+1}\right)\right)$; if there exists $n_{0}$ which $c_{n_{0}}=0$, then $f\left(x_{n_{0}}\right)=f\left(x_{n_{0}+1}\right)=g\left(x_{n_{0}}\right)$; therefore, $x_{n_{0}} \in S$ and the theorem is proved. On the contrary, we will have $c_{n}>0$ for each $n$, and, at the first time, we are going to prove $c_{n}>0$. Since $0<c_{n}$, (i). implies $c_{n}<d\left(g\left(x_{n}\right), g\left(x_{n+1}\right)\right)=$ $c_{n+1}$; the sequence is decreasing and positive. Let $c=\lim _{n} c_{n}$. If $c>0$, (i) implies that there exists a positive number $\delta$ such that, taking $c_{n}$ that keeps $c_{n}<c+\delta$ we have

$$
c \leq c_{n}<c+\delta \Rightarrow c_{n+1}<c \quad \text { and it contradicts }
$$

$c_{n} \vee c$; therefore, $c_{n}>0$ as we would like to see.

Now, we are going to prove that $f\left(x_{n}\right)$ is a Cauchy sequence. The proof is by contradiction. If $f\left(x_{n}\right)$ wasn't a Cauchy sequence there would exist $\varepsilon>0$ so that Cauchy relation wouldn't keep for $2 \varepsilon$. For a such $\varepsilon$, 
there exists $\delta>0$ keeping (i). For a such $\delta$ pick s so that

$$
c_{s}<\delta / 3
$$

Pick $k, m>s$ fulfilling $d\left(f\left(x_{k}\right), f\left(x_{m}\right)\right)>2 \varepsilon$ and $k<m$. If $n \in[k, m]$,

$$
\left|d\left(f\left(x_{k}\right), f\left(x_{n+1}\right)\right)-d\left(f\left(x_{k}\right), f\left(x_{n}\right)\right)\right|<\delta / 3 .
$$

Consider $A=\left\{n \in[k, m]: d\left(f\left(x_{k}\right), f\left(x_{n}\right)\right) \geq \varepsilon+\delta\right\}$, obviously $m \in A$, and let $i \in[k, m]$ be such that $i+1=\min A$. We have

$$
d\left(f\left(x_{k}\right), f\left(x_{i+1}\right)\right)-d\left(f\left(x_{k}\right), f\left(x_{i}\right)\right) \geq \varepsilon+\delta-d\left(f\left(x_{k}\right), f\left(x_{i}\right)\right)
$$

and, (3) and (4) imply $d\left(f\left(x_{k}\right), f\left(x_{j}\right)\right)>\varepsilon+2 \delta / 3$.

However, $d\left(f\left(x_{k}\right), f\left(x_{j}\right)\right) \leq d\left(f\left(x_{k}\right), f\left(x_{k+1}\right)\right)+d\left(f\left(x_{k+1}\right), f\left(x_{i+1}\right)\right)+$

$$
+d\left(f\left(x_{i+1}\right), f\left(x_{j}\right)\right)<E+2 \delta / 3
$$

because of (2), $i \notin A,(5)$ and (i). It contradicts (5). This contradiction proves that $f\left(x_{n}\right)$ must be a Cauchy sequence.

$$
\text { Because of (iv), } f\left(x_{n}\right) \rightarrow y \in \overline{g(x)} \text {. Let } B=\{y\} \cup\left\{f\left(x_{n}\right)\right\}_{n} \in N
$$

which is a compact of $Y$. (ii) implies that $f^{-1}(B)$ is compact; therefore, there exists a partial sequence of $x_{n}$ converging to $x \in f^{-1}(B)$, and $f(x)=y$ by the continuity of $f$. The continuity of $g$, given by (i), implies that the image by $g$ of this partial converges to $g(x)$; moreover, the handling of the sequence $x_{n}$ inplies $g(x)=f(x)$ and $S$ is non-empty as we would like to prove. The theorem is proved.

COROLLARY. If we change $f$ proper by $g$ proper between the hypothesis of the theorem I, this one will remain true. 
EXAMPLE. Let $x=[0,1\} \cup\{3 n, 3 n+1\}_{n \in \mathbb{N}}$ ard $Y=\mathbb{T}^{2}$, sot with e tclidean distance, and let

$$
f(x)=(x / 2, \sqrt{3} / 2 x)
$$

$$
g(x)= \begin{cases}(x / 4, \sqrt{3} / 4 x) & \text { if } x \in[0,1] \\ (0,0) & \text { if } x=3 n \\ (1 / 2-1 / 2 n+4, \sqrt{3} / 2-\sqrt{3} / 2 n+4) & \text { if } x=3 n+1\end{cases}
$$

There isn't any mapping $\varphi$ such that $d(g(x), g(y)) \leq \varphi\left(d\left(f^{\prime}(x), f(y)\right)\right)$ kecping $\varphi(\varepsilon)<\varepsilon$ for each $\varepsilon>0$ because $\varphi(1)$ couldn ${ }^{\prime} t$ be minor than 1 since $d(f(3 n), f(3 n+1))=1$ and $d(g(3 n), g(3 n+1))=1-1 / n+2$, arid $\varphi$ should keep $1-1 / n+2 \leq \varphi(1)$ for each $n$. Conversely, we can prove without any difficulty that these mappings keep the hypothesis of theorem I.

\section{THEOREM II}

THEOREM II. Let $(X, U)$ be a non-empty Hausdorff complete uniform space, $f$ and $g$ be two functions from $x$ into $x$. If

(i) $f$ is uniformly continuous of $\left(x, U_{\varphi}\right)$ into $(x, u)$

(ii) $\forall u \in U$ JV $\in=$ such that $(f(x), f(y)) \in V \Rightarrow(x, y) \in U$

(iii) $\varphi^{-1}(U)$ is non-empty and closed for each closed symmetric member $U$ of $U$.

Then $f$ and $g$ have a unique coincidence point.

Proof. Pick the filter $F=\left\{\varphi^{-1}(U !: U \equiv E\}\right.$, where $B$ is the set formed by all the closed symmetric members of $U$.

We will see $F$ is a Cauchy filter. let $U \in U$, pick $V \in U$ such that $(f(x), f(y)) \in V \Rightarrow(x, y) \in U$ since (ii). By (i) we can find $W \in B$ such trat $(x, y) \in \varphi^{-1}(w) \times \varphi^{-1}(w) \cup \Delta \Rightarrow(f(x), f(y)) \in V$. Taking $\varphi^{-1}(w)$ we have $\varphi^{-1}(W) \in F$ and $\varphi^{-1}(W) \times \varphi^{-1}(W) \subset U$; therefore $F$ is a Cauchy filter. 
Since $F$ is a Cauchy filter, $X$ complete Hausdor $f$ ino ( $i i_{1}$, we have

$$
\cap_{w \in B} \phi^{-1}(w)=\left\{x_{0}\right\}
$$

inoreaver, taking images by $\varphi$ it results that $A=\underset{W \in B}{\cap} W\left(f\left(x_{0}\right), g\left(x_{0}\right)\right.$ impiias $f\left(x_{0}\right)=g\left(x_{0}\right)$. We shall now prove the unicity. If $y_{0}$ is a coincidence point $\varphi\left(y_{0}\right) \in \Delta \Rightarrow \varphi\left(y_{0}\right) \in \underset{W \in B}{n} W \Rightarrow y_{0} \in \underset{W \in E}{\cap} \varphi^{-1}(W)=\left\{x_{0}\right\} \Rightarrow y_{0}=x_{0}$ and the theoren is proved.

LOROLLARY. If $X$ is a complete metric space, the theorem says:

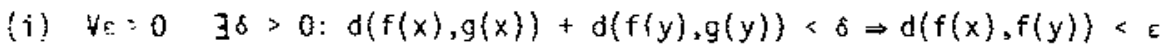

(ij) $V_{\varepsilon}>0 \quad \exists \delta^{\prime}>0: d(f(x), f(y))<\hat{o}^{*} \Rightarrow d(x, y)<\varepsilon$

(iji) $\forall \varepsilon=0 \quad x: x \in X$ and $d(f(x), g(x)) \leq \varepsilon\}$ is non-empty and closed.

Then, $f$ and $g$ have a unique coincidence point.

Proof. It is enough to observe that a vecinity of $v_{\varphi}$ has the form $1 l^{\prime}=\{(x, y): x=y$ or $\{d(f(x), g(x)\}<r$ and $d(f(y), g(y))<r]\}$ and if we consider $B_{\varphi}^{r}=\{(x, y): x=y$ or $d(f(x), g(x)) \neq d(f(y), g(y))<r\}$ we will have $B_{\varphi}^{r} \subset u_{\varphi}^{r} \subset B_{\varphi}^{2 r}$.

\section{REFERENCES}

1. J.L. Cerdã Martín: A coincidence theorem. Oubi. Mat. U.A.8.7.

2. A. Meir - E. Keelor: A theorem on contraction mafpings. Journal of Math. Analysis and Apl. 28, 326-9 (69).

3. Shi Song Wong: A fixed point theorem for a class of mapping. Math. Ann. $204(3973) ; 97-103$.

Aquest treball fou presentat a les Jornades lill de Santander (1979). 